\title{
Houssay, Leloir y los nóbeles latinoamericanos de ciencias
}

\author{
Alfredo Jácome Roca*
}

Internista-Endocrinólogo, miembro honorario de la Asociación Colombiana de Endocrinología, Diabetes y Metabolismo, Miembro de Número de la Academia Nacional de Medicina, y Miembro Activo de la Sociedad Colombiana de Historia de la Medicina.

Es el Editor de la obra "Fisiología Endocrina".

Fecha de recepción: 2/02/2017

Fecha de aceptación: 13/02/2017

\section{Resumen}

$\mathrm{L}$ a ciencia latinoamericana tuvo un auge con los investigadores del Cono Sur, en particular argentinos, siendo tres de ellos galardonados con el premio Nobel. El primer maestro fue Bernardo Houssay quien, con su discípulo Luis Federico Leloir, logró consolidar un grupo de investigadores en temas hormonales, que tuvieron resonancia mundial. La ciencia fue progresivamente dirigiéndose a la moderna biología molecular. Infortunadamente, las crisis políticas y económicas fueron alejando a muchos, a pesar del reconocido nacionalismo de los argentinos. Actualmente, la producción científica latinoamericana es marginal en comparación con Estados Unidos y los países europeos, y aunque se han venido formando numerosos científicos gracias a los programas de doctorado y al buen recurso humano, los problemas políticos y los dudosos manejos estatales de los escasos ingresos, no han permitido un nuevo despegue de la investigación regional.

Palabras clave: ciencia, tecnología, innovación, competitividad, fisiología, bioquímica, metabolismo, premio Nobel, política científica.

\begin{abstract}
Science in Latin America had a peak with southern cone researchers, particularly the Argentinian ones, being three of them awarded with a Nobel prize in sciences. First master was Bernardo Houssay who, along with his pupil Luis Leloir, consolidated a group of scientists that digged into the mystery of hormones and metabolism, reaching world fame throughout time. Science then moved to modern molecular biology.

Unfortunately political and economic crises led some to migrate, independently of known Argentinian nationalism. Nowadays, science production in Latin America is marginal, as com-
\end{abstract}

pared to United States and some European countries. Although, thanks to government programs to generate investigators with doctorates we have more qualified human resources, political problems and doubtful expenditure of scarce incomes have not allowed a definitive launching in regional research

Keywords: Science, technology, innovation, competitiveness, physiology, biochemistry, metabolism, Nobel Prize, science policies.

\section{Aportes científicos latinoamericanos}

Dos científicos suramericanos fueron ganadores en el siglo XX de sendos premios Nobel por sus trabajos sobre el metabolismo hidrocarbonado: Bernardo A. Houssay (Fisiología y Medicina) y Luis Federico Leloir (Química). A pesar de la polarización política que ha vivido la República Argentina desde los tiempos del general Perón, aquellas épocas fueron luminosas para la ciencia del país austral, gracias a las investigaciones realizadas por estos dos brillantes estudiosos, cuyo tesón logró superar las limitaciones existentes ${ }^{(1)}$.

Actualmente, los científicos más creativos de muchas naciones, migran a Estados Unidos o al Reino Unido, donde se ubican las grandes universidades que tienen los recursos, la infraestructura y la logística para desarrollar proyectos de gran envergadura. Se trata además de países políticamente estables.

En ese mar de norteamericanos y europeos ganadores de algún premio Nobel en ciencias, solo pocos latinoamericanos han navegado. Aparte de los ya nombrados, tres más lo han logrado, dos de ellos en el campo de la inmunología. Pero migraron para desarrollar sus proyectos. Se trata del venezolano Baruj Benacerraf (1920-2011), quien desde joven se formó profesionalmente en los Estados Unidos e hizo su investigación sobre antígenos de histocompatibilidad; y el también argentino César Milstein (1927-2002), por el Reino Unido, con estudios sobre anticuerpos monoclonales. De este último hay que anotar que nació, realizó sus estudios formales y además obtuvo su primer doctorado en la Argentina, pero su vida cambió al ser becado en Cambridge para trabajar en el laboratorio de Frederick Sanger. Este destacado ciudadano inglés obtuvo lauros de Estocolmo, tanto en química como en fisiología y medicina; fue él quien definió la estructura de la insulina. En 
un plan retorno a su país, en 1961, para hacerse cargo de la División de Biología Molecular del Instituto Nacional de Microbiología, Milstein solo estuvo un año en el cargo para regresar a Inglaterra tras el golpe militar de 1962. Cambridge fue su hogar desde ese entonces, y sus importantes descubrimientos fueron donados a la humanidad (se negó a patentarlos y hacerse rico), debido a su ideario esencialmente científico y humanista. En su juventud fue algo rebelde y había militado en un movimiento anarquista.

Adicionalmente aparece un investigador mexicano, Mario Molina, quien recibió el Nobel de Química por su trabajo sobre la formación y descomposición del ozono, pero su labor científica fue realizada en California.

Los primeros cuatro científicos tenían padres de diferentes nacionalidades: Houssay y Leloir (de origen francés), Milstein (judíos ucranianos) y Benacerraf (marroquíes sefarditas). Paul, hermano menor del último, era un filósofo y matemático nacido en Francia y radicado posteriormente en los Estados Unidos. Pero ¿no sabemos luego que América Latina era indígena, y que las migraciones europeas y medio-orientales vinieron a incrustarse en sus genes? Solo que algunas familias estuvieron muy de paso $^{(2,3)}$.

\section{La ciencia argentina crece con Houssay}

Bernardo A. Houssay nació el 10 de abril de 1887 en Buenos Aires y murió en la misma ciudad el 21 de septiembre de 1971. Este médico y fisiólogo argentino fue un niño prodigio, salió de bachiller a los 13 años y a los 23 ya ostentaba dos títulos universitarios: un grado en farmacia y el de médico, ambos de la Universidad de Buenos Aires. A esa corta edad fue nombrado docente de fisiología en la Facultad de Veterinaria ${ }^{(4)}$.

Houssay era clínico, y ejerció privadamente la medicina. Dirigió incluso dos servicios en hospitales bonaerenses. Pero al tiempo dirigió la patología experimental en el Instituto de Salud Pública de la capital. Alguna vez tuvo relación con un caso de acromegalia originada en un tumor pituitario, lo que le interesó en sumo grado; por esto dirigió su interés hacia la hipófisis, llevándolo a abandonar el ejercicio profesional y a formarse como investigador ${ }^{(5)}$.

En 1911 obtuvo su doctorado con una tesis sobre la función de la hipófisis, y en 1919 fue nombrado Jefe del Departamento de Fisiología en la Escuela de Medicina de la Universidad de Buenos Aires. Aquello se volvió un centro de investigación que atrajo y formó algunas de las figuras científicas más influyentes de América Latina. Cerca de un millar de publicaciones de Houssay cubrieron todo el espectro de la fisiología humana: las glándulas endocrinas, el aparato respiratorio, el sistema circulatorio, el nervioso y el inmune. A pesar de su inteligencia, imaginación, destrezas y conocimiento, se trató de una persona sencilla y humilde; era un fisiólogo de verdad que nos hace sonrojar cuando escribimos textos sobre el tema, pues muchos no somos más que clínicos informados de la literatura ${ }^{(6)}$.

Desde sus estudios de posgrado, fue un ávido lector de los trabajos del fisiólogo Claude Bernard, y sabía de los perros pancreatectomizados de Minkowski, que terminaron diabéticos por este procedimiento. Luego vinieron los experimentos de Banting y Best, quienes en 1921 descubrieron la insulina. Lo suyo era la hipófisis: quería conocer las funciones fisiológicas de los extractos pituitarios que en aquella época se relacionaban más con los efectos antidiuréticos e hipertensivos de la vasopresina. Incorrectamente se creía en aquel entonces que algún efecto que pudiese tener la hipófisis sobre el metabolismo de los carbohidratos se debía al lóbulo neural. Metódico como era, Houssay coordinó sus esfuerzos en relación con el metabolismo hidrocarbonado, revisando primero toda la literatura disponible al respecto, descartando algunas cosas y enfocándose en otras. Se entrenó en el cultivo de estos tejidos y en su análisis, para aislar los compuestos fisiológicamente activos. Tal vez por alguna correspondencia con el doctor Bernard Aschner (tío abuelo del endocrinólogo colombiano Pablo Aschner), aprendió su técnica de hipofisectomía que había llevado a aquel a realizar importantes experimentos sobre la relación de la pituitaria anterior y los ovarios ${ }^{(2)}$.

Observó el efecto diabetogénico de las hormonas contrarreguladoras de la insulina, notando la mejoría del perro diabético pancreatectomizado al realizar hipofisectomías, disminuyéndose de esta forma sus requerimientos de insulina. Houssay constituyó un equipo de trabajo para investigar la acción de la insulina. Entre otros resultados, halló que los perros a los que se había extirpado la hipófisis tenían reacciones hipoglucémicas muy acentuadas luego de la inyección de pequeñas cantidades de insulina. Este hecho inesperado lo hizo intentar en el perro la doble extirpación de la hipófisis y el páncreas, comprobando -con profunda sorpresa- que los perros que carecían de páncreas y de hipófisis no se tornaban diabéticos. Este descubrimiento acaeció en 1929. El fisiólogo había descubierto que la diabetes pancreática no se producía en dichos perros hipofisoprivos. El inglés Young había mostrado que el uso prolongado de extracto hipofisiario terminaba causando diabetes permanente en el animal, aun en aquellos con páncreas intactos. El argentino había trabajado en la misma dirección. Tampoco se producía en los sapos en los cuales, dada la conformación histológica de la glándula, era posible extirpar por separado el lóbulo glandular, equivalente a la parte anterior de la hipófisis de los mamíferos y aislándola de la neurohipófisis. En los sapos, la diabetes reaparecía con el injerto de lóbulos glandulares ${ }^{(7)}$.

Finalmente, realizó con éxito otra contraprueba: la inyección de extractos de la parte anterior de la hipófisis provocaba hiperglucemia. La homeostasis de la glucemia no dependía entonces solo de la insulina. Consideró que la hipófisis anterior contenía una hormona a la que llamó "factor diabetógeno"(2). 
¿Cómo actuaba ese extracto? ¿Se trataba de un efecto aloxano como lo postuló el inglés Young en 1937? Caló más la hipótesis de Cori, quien afirmó que dicho extracto hipofisiario usado por Houssay inhibe la enzima hexoquinasa, tanto in vivo como in vitro, inhibición que es abolida por la insulina.

Estos hallazgos tuvieron un impacto clínico, probablemente infortunado. Durante varios lustros, los pacientes con diabetes no controlada que empezaban a desarrollar retinopatía eran sometidos a hipofisectomía. Pero a cambio debían afrontar un hipopituitarismo de por vida, con la desventaja de que en aquellas épocas no se contaba con las terapias de suplencia con hormonas manufacturadas con métodos de ingeniería genética por ADN recombinante. Tuve la oportunidad de ver en la década de los 60 en la Universidad de Tulane un paciente de estos, que se había complicado con una profusa rinorragia.

El profesor Houssay amaba a su patria e intentó hacer que la ciencia hablara castellano. Fue maestro de muchos, pero no tuvo maestro, pues fue autodidacta. Sus publicaciones fueron hechas en español, en revistas argentinas. Los americanos demoraron años en entender sus estudios, solo hasta que se realizaron cuidadosas traducciones al inglés de aquellos artículos científicos.

\section{¿Cuáles debían ser los objetivos científicos de la inversión?}

En aquellas épocas de economías cerradas y radicalismos, los científicos no estuvieron exentos de enfrentamientos. Había unos que exigían la autorregulación, que debían ser libres en los temas a investigar -la ciencia por la ciencia-y otros que clamaban por recursos invertidos únicamente en campos que llevaran al desarrollo social y económico, lo que debía constituirse en política pública de ciencia y tecnología. Aunque se consideraba que a comienzos de los años cuarenta Argentina estaba a punto de convertirse en país desarrollado, no lo era aún, y los dineros estatales debían gastarse en la solución de problemas vinculados a las áreas de energía, salud, recursos naturales, producción o defensa.

\section{La situación política}

Los líderes carismáticos, populistas y nacionalistas nacen de la inconformidad de la gente con las políticas liberales, expresadas en gobiernos débiles. Aparecen los autoritarios, con ideas propias sobre cómo debe imponerse la justicia social con el embeleco de que quien debe mandar es el pueblo. El máximo líder sabe -sin temor a equivocarse- cuáles son las medidas que contra viento y marea impondrá, por la fuerza si es necesario. Esos tiempos oscuros se impusieron por la vía electoral en aquella época de Alemania, con un ideario nacional socialista y anticomunista, pero también en la Argentina, por la vía del golpe militar. El gobierno austral germanófilo hizo que allí terminaran jefes nazis (¿con sus fortunas?) que tuvie- ron finales distintos ante la posterior búsqueda y persecución de los sabuesos sionistas ${ }^{(9-11)}$. El médico Joseph Mengele ("El ángel de la Muerte") logró escapar a Brasil. Otros no tuvieron esa suerte. Los más, terminaron sus días en el anonimato, aunque seguían realizando nostálgicas fiestas para rememorar el poder nazi que alguna vez tuvieron.

La ideología peronista está llena de lugares comunes, de frases difusas en donde abundan palabras como pueblo, justicia, partido, equidad, orden, trabajo, unidad, interés social. Pero desaparece un vocablo esencial: libertad. El líder carismático que pronto mostrará su autoritarismo ha logrado encaramarse. El pueblo seguirá sus órdenes sin chistar, porque su populista jefe es el único que sabe lo que le conviene. Los tiempos se reciclan. Cae como anillo al dedo el dicho de que quien ignora la historia, está condenado a repetirla ${ }^{(9-15)}$.

Así fue como en 1943, después del golpe militar de Perón (1895-1974) y su gobierno de facto, un enamorado de la libertad como Houssay fue retirado de su puesto, y aunque por su prestigio se le readmitió dos años después, de nuevo lo sacaron. A otros 150 intelectuales les pasó lo mismo. Ese fatídico 1943, en plena Segunda Guerra Mundial en el que el fisiólogo argentino firmó la carta contra el régimen nazi de Alemania y el apoyo del general Ramírez (y de su golpista sucesor el general Perón), exilió de la Argentina a su segundo Nobel de Fisiología y Medicina, el médico y bioquímico Luis F. Leloir, su alumno más destacado, quien se radicó por un par de años en los Estados Unidos. Otros discípulos también insignes fueron Juan Carlos Fasciolo, Juan Muñoz, Alberto Taquini, Braun Menéndez ${ }^{(8)}$, su hijo Alberto Houssay, Lewin, Óscar Orías, Hug y Foglia, algunos de ellos estudiosos de los aspectos endocrinos de la hipertensión y descubridores de la angiotensina; otros fueron continuadores de su opera magna, la "Fisiología Humana", que logró muchas ediciones (al menos siete) y traducciones a varios idiomas. Su editorial fue la recordada "El Ateneo", que también honró al autor de este artículo con la publicación en Buenos Aires de las dos primeras ediciones de su "Fisiología Endocrina” y que se apresta en 2017 a publicar la cuarta edición, con la colaboración de más de 20 endocrinólogos colombianos. Virgilio Foglia (1905-1993) es recordado como investigador de la secreción insulínica, fundador del IBYME, de la Sociedad Argentina de Endocrinología y de la Sociedad Argentina de Diabetes. Fue Presidente de la Academia Nacional de Medicina (1986-88) y de la Sociedad Argentina de Ciencias Fisiológicas (1971-78). También fueron pupilos de Houssay (y de Leloir) Rangel Capito, Alejandro Paladina, Carlos Cardini y Raúl Truco, que con Leloir trabajaron en el Instituto Campomar de Investigaciones Bioquímicas.

Varios amigos del Profesor se comunicaron con sus contactos en el extranjero para que retomara su impulso en lares foráneos ${ }^{(9-11)}$. Pero él mostró sus intenciones de quedarse, en una carta dirigida a un colega de la Universidad de Harvard en la que escribió: 
"Mi intención es seguir trabajando en fisiología. He recibido numerosas invitaciones para trabajar en otros países. Sin embargo, mis planes de toda la vida no han cambiado; quiero trabajar duro en investigación para crear en mi país un círculo de científicos de la más alta moral y calidad científica. No cambiaré esta línea de conducta". Era necesario retener el talento nacional.

A pesar de la catástrofe que parecía un abrupto y prematuro final, esto no sucedió. Sus amigos se unieron y consiguieron fondos para que el profesor siguiera sus labores en un instituto privado. En 1944 fundó el Instituto de Medicina Biológica y Experimental (IBYME), que dirigió desde 1946. Analizó la acción del veneno de las serpientes y los arácnidos, así como diversos aspectos relacionados con la farmacología, la nutrición, la patología experimental y la pedagogía médica. En Norteamérica se creó el Houssay Journal Fund por los doctores Herbert Evans, Walter Cannon, John Fulton y Carl Wiggers, quienes consiguieron donaciones para comprar subscripciones de costosas y prestigiosas revistas científicas y, además, libros actualizados. Quien inició esta campaña fue la joven francesa Christiane Dosne, primera becaria del fisiólogo, quien se casó con el endocrinólogo Rodolfo Pasqualini y luego se convirtió en su principal biógrafa(10).

El proceso de fragmentación política e ideológica hizo impacto en la conformación institucional de la ciencia y tecnología; la intensidad y la persistencia del enfrentamiento político mantuvieron vigente la polarización a lo largo de por lo menos cuatro décadas. Muerto Perón -y su carnal Evita- el populismo peronista (fascismo en otros lares) aún persiste. La inconformidad e impaciencia porque las metas se demoran, inciden en el centro que se fragmenta y radicaliza en polos opuestos, haciendo funcionar la ley del péndulo. En Europa se pagó con una guerra que terminó con cincuenta millones de muertos. En América estamos viendo pobreza, desesperanza, autoritarismo, corrupción e incertidumbre, tanto más grave cuanto más poderoso es el país. Militares, ex militares y otra vez militares, metidos de políticos, o políticos apoyados en el vaivén del momento, aparecen de pronto con ímpetu mesiánico.

\section{Vienen los premios y crece el prestigio internacional}

En 1947 recibió el premio Nobel de Medicina y Ciencias Fisiológicas que compartió con Carl Ferdinand Cori y Jerry Teresa Cori, por el descubrimiento de la función hormonal de la hipófisis (figura 1). Los esposos Cori -quienes estudiaron la absorción y metabolismo de los azúcares- habían propuesto que la síntesis del glucógeno era realizada por la enzima glucógeno fosforilasa; pero fue el argentino Luis Federico Leloir quien demostró que se trataba más bien de la glucógeno sintasa o sintetasa.

Houssay tenía 24 doctorados honoris causa, era miembro de numerosas academias de medicina y de más de 200 socie-
Figura 1. Estampilla conmemorativa del Nobel de Houssay en 1947

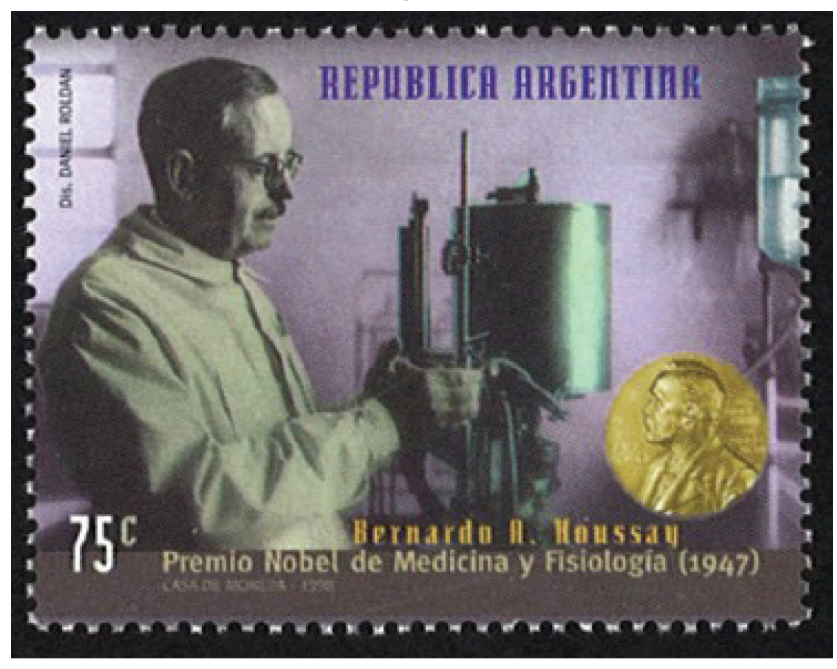

dades científicas. Las distinciones provinieron de instituciones tan famosas como Harvard, Cambridge, Oxford y la Universidad de París. Obtuvo la medalla Dale en 1960, considerada el más alto honor en el campo de la endocrinología. En 1972, la Organización de Estados Americanos instituyó el premio Bernardo A. Houssay, que se otorga anualmente a un investigador científico latinoamericano. Es muy conocida su obra Fisiología Humana, texto con el que estudiaron muchos médicos en la región.

Sus principales biógrafos, Rodolfo Q. Pasqualini y su esposa Christiane Dosne Pasqualini, afirmaron que le daban a Houssay tanta o más importancia por sus logros como político de la ciencia que como investigador. Destacaron la creación del CONICET (Consejo Nacional de Investigaciones Científicas y Técnicas), en 1958, como coronación de tanta lucha para asegurar el progreso de la ciencia en su país. Houssay fue el primero en presidirlo, hasta su muerte ${ }^{(16-18)}$.

\section{Luis Federico Leloir}

A diferencia de Houssay, cuyos padres fueron pobres, su discípulo más destacado era de antepasados terratenientes. Fue hijo póstumo y el menor de nueve hermanos, nacido en París (1906) adonde su padre había ido a operarse. Murió en Buenos Aires (1987). Creció en la pampa argentina en las extensas tierras de la familia, que contaban con cuarenta mil hectáreas. Aprendió a leer solo y su lectura era sobre temas agropecuarios que traían los periódicos. En mi primera visita a Buenos Aires (1973) me sorprendió que el diario traía dos grandes secciones, la segunda exclusivamente dedicada al campo y a la ganadería. En tiempos de la segunda guerra, Argentina era el primer exportador de carne del mundo, superado décadas más tarde por Australia. Se convirtió en acucioso observador de los fenómenos naturales, y siempre prefirió lecturas acerca de las ciencias naturales ${ }^{(19-23)}$. 
Estudió medicina en la Universidad de Buenos Aires y fue residente en el famoso centro médico paulista, el Hospital Das Clinicas. Dejó la medicina para dedicarse a la investigación. Conoció a Houssay a través de parientes políticos, y este le dirigió su tesis de grado sobre la influencia de las glándulas suprarrenales en el metabolismo de los carbohidratos. Esto marcó su campo investigativo en la bioquímica que se centró en los nucleótidos de los glúcidos, estudios que ayudaron en la comprensión de la galactosemia congénita.

Trabajando con su maestro Houssay, entendió que su formación en ciencias era escasa, por lo que se convirtió en "alumno oyente" de las clases de física, química, biología y matemáticas en la misma universidad que le había expedido el título de médico. Los profesionales que hace décadas estudiamos medicina, estábamos familiarizados con la figura del "asistente a clases", una persona a quien los catedráticos permitían el ingreso por su solo interés de informarse y aprender. En 1936 viajó a Cambridge (la misma universidad donde años más tarde trabajaría Milstein) para especializarse en bioquímica de carbohidratos, bajo la supervisión de Frederick G. Hopkins (Nobel de Fisiología y Medicina en 1929 por sus estudios en vitaminas).

Regresó a Buenos Aires al lado del maestro, pero con el golpe peronista inmigró a los Estados Unidos donde investigó en prestigiosas universidades y adquirió destrezas en el tema de la enzimología. De nuevo retornó a su patria dos años más tarde, para vincularse al instituto privado que dirigía Houssay. Allí experimentó sobre las rutas químicas en síntesis de azúcares con equipos baratos y poco sofisticados, sugiriendo que las mentes brillantes no requieren tecnologías de punta, como ocurrió con descubridores científicos del siglo XIX.

Su grupo productivo de trabajo incluyó otros discípulos de Houssay que ya hemos mencionado. Allí se hicieron investiga- ciones sobre el papel del riñón en la hipertensión, se identificaron los carnucleótidos y la uridina difosfato glucosa (UDPG) que ayudó a entender el proceso de almacenamiento de los carbohidratos y su transformación en energía de depósito.

A comienzos de la segunda mitad del siglo XX, la Fundación Rockefeller y el Massachusetts General Hospital le ofrecieron establecerse allí para continuar sus investigaciones. A pesar de que rechazó la jugosa oferta, ellos y el Instituto Nacional de Salud de los Estados Unidos decidieron subsidiar sus trabajos, por considerarlos de suma importancia. Continuó estudiando la síntesis hepática de glucógeno y la oxidación de ácidos grasos con extractos celulares de hígado.

Leloir fue Nobel de Química en 1970; su investigación más relevante se centró en los nucleótidos de azúcar, el papel que cumple en la fabricación de los hidratos de carbono y la comprensión pormenorizada de la galactosemia ${ }^{(20-23)}$. Su equipo se dedicó a estudiar las glucoproteínas. Al igual que Houssay, recibió numerosos títulos y distinciones en diversas partes del mundo. Pero prefirió continuar ayudando en la construcción de la reconocida escuela argentina de diabetes y nutrición, en la que nuestro pionero Mario Sánchez Medina se formó inicialmente, y quien intercambió correspondencia con el profesor Houssay y los demás científicos australes. No en vano recibiría años más tarde el premio Hagedorn por su investigación en diabetes.

A pesar de estos excelentes antecedentes, pareciera que lo importante en América Latina fuera el fútbol, la farándula o la política. La honestidad y otros profundos valores de estos pioneros han venido siendo remplazados por la ambición, la corrupción y la consecución, bien del dinero fácil o producido por actividades legales pero que distan mucho de las intelectuales. ¡La humanidad le debe tanto a tan pocos!

\section{Referencias}

1. Von Stecher P. Hacia una medicina argentina: ciencia, nación y escritura. Arch Med (Manizales) 2016, 16(1):167-77.

2. Jácome A. Historia de las Hormonas. Academia Nacional de Medicina, Bogotá. 2005.

3. Jácome A. Historia de los Medicamentos. Academia Nacional de Medicina, Bogotá 2003.

4. Pasqualini CD. Arte y política de la ciencia. Bernardo A. Houssay y Harold Varmus. Medicina (B. Aires) 2009; 69 (6).

5. Tan SY, Ponstein N. Bernardo Houssay (1887-1971): Endocrine physiologist and Nobel laureate.

6. Zetterström R. C. Cori (1896-1984), G. Cori (1896-1957) and B. Houssay (1887-1971). Nobel Prize in 1947 for discoveries of glycogen metabolism with relevant and irrelevant clinical implications. Acta Pædiatrica, 2007; 96 : 935-938.

7. Houssay BA. The Nobel Foundation. Nobel Lecture: The Role of the Hypophysis in Carbohydrate Metabolism and in Diabetes [online] [Accessed January 25, 2017]. Available at: http://nobelprize.org/nobel_prizes/medicine/ laureates/1947/houssay-lecture.html

8. Braun Menéndez E, Fasciolo JC, Leloir LF, Muñoz JM, Taquini AC. Hipertensión arterial nefrógena. Buenos Aires: El Ateneo, 1943.

9. Pasqualini CD. El significado del Premio Nobel en Fisiología o Medicina. Medicina (Buenos Aires) 2007; 67: 309-13.

10. Pasqualini CD. Bernardo A. Houssay (1887-1971) Medicina (Buenos Aires) 2003; 63: 90-1.

11. Stoppani AOM. Centenario del nacimiento de Bernardo A. Houssay. Colección Academia Nacional de Medicina, 1987; 6: 97-108.

12. Pasqualini RQ. Houssay y el serendipismo. Medicina (Buenos Aires) 1981; 41: 827-30.

13. Pasqualini RQ. La formación de un fisiólogo. Medicina (Buenos Aires) 1987; 47: 681-5.

14. Pasqualini RQ. Houssay y la investigación biomédica. Medicina (Buenos Aires). 1997; 57: 635-6.

15. Pasqualini CD. Houssay y el Premio Nobel. Medicina (Buenos Aires) 1997; 57: 637-40.

16. Pasqualini CD. 1944: Houssay y el nacimiento del IBYME. Medicina (B. Aires) 2014; 74: 216-219.

17. Hurtado D, Fernández MJ. Institutos privados de investigación "pura" versus políticas públicas de ciencia y tecnología en la Argentina (19431955). Asclepio, 2013; 65 (1): p010 2013. http://dx.doi.org/10.3989/asclepio.2013.10.

18. Charreau EH. Relato histórico del Instituto de Biología y Medicina Experimental. Acta Bioquim Clin Latinoam 2011; 45: 599-713.

19. Barañao L. ¿Hacia dónde va la ciencia argentina? Medicina (B Aires) 2012; 72: 339-49.

20. Pasqualini CD. [Science in 1983 according to Luis Federico Leloir. Comparison with the current situation according to Lino Barañao]. Medicina (B Aires). 2014; 74(4):340-1.

21. Parodi AJ. Luis Federico Leloir, or how to do good science in a hostile environment.IUBMB Life. 2012; 64(6):567-72.

22. Pasqualini CD. El destino de los investigadores en ciencia. Medicina (B Aires) 2013; 73:179-82.

23. Pasqualini CD. Setenta años de investigación en biomedicina del Canadá a la Argentina. Ciencia e Investigación Reseñas 2013; 1: 22-38. 